\title{
NILPOTENCE AND TORSION IN THE COHOMOLOGY OF THE STEENROD ALGEBRA
}

\author{
KENNETH G. MONKS
}

\begin{abstract}
In this paper we prove the existence of global nilpotence and global torsion bounds for the cohomology of any finite Hopf subalgebra of the Steenrod algebra for the prime 2. An explicit formula for computing such bounds is then obtained. This is used to compute bounds for $H^{*}\left(\mathscr{A}_{n}\right)$ for $n \leq 6$.
\end{abstract}

\section{INTRODUCTION}

Let $R$ be any commutative algebra and $x \in R$. An element $y \in R$ is said to be $x$-torsion if $x^{k} y=0$ for some $k . R$ has $x$-torsion bound $m$ if $x^{m} y=0$ for any $x$-torsion $y \in R . R$ has global torsion bound $m$ if $m$ is an $x$-torsion bound for all $x \in R$. $R$ has global nilpotence bound $m$ if $x^{m}=0$ for all nilpotent $x \in R$. For any algebra $R, x \in R$ has nilpotence $k$ if $x^{k}=0$ and $x^{k-1} \neq 0$.

Let $\mathscr{A}$ be the Steenrod algebra at prime 2. Let $\mathscr{A}\left(n_{1}, n_{2}, \ldots\right)$ be the $\mathbb{Z}_{2}$ submodule of $\mathscr{A}$ generated by the Milnor basis elements $S q\left(r_{1}, r_{2}, \ldots\right)$ with $r_{i}<2^{n_{i}}$ for all $i$. If $\exists k$ such that $n_{i}=0$ for all $i>k$ then we will write $\mathscr{A}\left(n_{1}, \ldots, n_{k}\right)$ for $\mathscr{A}\left(n_{1}, n_{2}, \ldots\right) . \mathscr{A}\left(n_{1}, n_{2}, \ldots\right)$ is a Hopf subalgebra of $\mathscr{A}$ if and only if $n_{v} \geq \min \left\{n_{v-u}, n_{u}+u-v\right\}$ for all $u, v$ with $v>u \geq 1$. Further, these are the only Hopf subalgebras of $\mathscr{A},[2,3]$.

Let $\Gamma=\mathscr{A}\left(n_{1}, \ldots, n_{k}\right)$ be any finite Hopf subalgebra of $\mathscr{A}$ and $H^{*}(\Gamma)=$ $\operatorname{Ext}_{\Gamma}\left(\mathbb{Z}_{2}, \mathbb{Z}_{2}\right)$. Our main results are

Theorem I.1. $H^{*}(\Gamma)$ has a global nilpotence bound which is computable by Proposition 1-3 below.

Theorem I.2. Any global nilpotence bound for $H^{*}(\Gamma)$ is also a global torsion bound (and hence an $x$-torsion bound for any element $x \in H^{*}(\Gamma)$ ) and vice versa.

Thus the global nilpotence bounds in Theorem I.1 are also global torsion bounds for $H^{*}(\Gamma)$. None of the bounds given above are known to be the best possible, i.e. there may be smaller bounds than those given. The following propositions are those referred to in Theorem I.1.

Proposition 1 (W. H. Lin [6]). $H^{*}(\Gamma)$ is nilfree if and only if

(a) $\Gamma=\mathscr{A}\left(0, \ldots, 0, n_{t}, n_{t+1}, \ldots\right)$ with $n_{i} \leq t \quad \forall i$, or

Received by the editors August 8, 1989 and, in revised form, August 23, 1990.

1980 Mathematics Subject Classification (1985 Revision). Primary 16A61, 55S10; Secondary 57T05, 55U99. 
(b) $\Gamma=\mathscr{A}\left(0, \ldots, 0,1, n_{t+1}, n_{t+2}, \ldots\right)$ with $n_{i} \leq t+1 \quad \forall i$ and $n_{j}=t+1$ for at least one $j$.

In this case, $H^{*}(\Gamma)$ trivially has global nilpotence bound 1 .

Proposition 2. Suppose $\Gamma$ is a finite Hopf subalgebra of $\mathscr{A}$; and suppose

(a) there exists a finite family $\left\{\Lambda_{j} \mid 1 \leq j \leq n\right\}$ of Hopf subalgebras of $\Gamma$, such that for each $j, \Gamma$ is obtained from $\Lambda_{j}$ by the addition of one generator whose class is $\alpha_{j}$ in the same sense of Definition II.1;

(b) there exist integers $w_{j} \geq 0$ such that $\prod_{j=1}^{n} \alpha_{j}^{w_{j}}=0$ in $H^{*}(\Gamma)$;

(c) $H^{*}\left(\Lambda_{j}\right)$ has global nilpotence bound $m_{j}$; then $H^{*}(\Gamma)$ has global nilpotence bound $\sum_{j=1}^{n} w_{j} m_{j}$.

In $\S$ II we will define the notion of a ' $k, m$-allowable' Hopf subalgebra of $\Gamma \subset \mathscr{A}$ (Definition II.4). To such a Hopf algebra $\Gamma$ we will associate Hopf subalgebras $\Gamma_{(k)}$ and $\Gamma_{(m)}$ (Definition II.3). We will also define a sense in which a Hopf algebra can be built up from a Hopf subalgebra by the addition of one generator whose class is $h_{s, t}$ ' (Definitions II.1 and II.2).

Proposition 3. For any $\Gamma$ and any integers $k, m$ such that $\Gamma$ is $k, m$-allowable, $\Gamma$ is obtained from $\Gamma_{(k)}$ (and also from $\Gamma_{(m)}$ ) by the addition of one generator whose class is $h_{n_{k}, k}$ (resp. $h_{n_{m}, m}$ ) and such that,

(a) $h_{n_{k}, k}^{2^{n_{m}-n_{k}-k}} h_{n_{m}, m}=0$ if $n_{m} \geq n_{k}+k$,

(b) $h_{n_{k}, k} h_{n_{m}, m}^{2^{n_{k}+k-n_{m}}}=0$ if $n_{m}<n_{k}+k$.

Further, if $H^{*}(\Gamma)$ is not nilfree then there exists at least one such pair of integers.

While there is always one pair of integers $k, m$ so that $\Gamma$ is $k, m$-allowable in Proposition 3 (unless $H^{*}(\Gamma)$ is nilfree), there are often several such pairs. The freedom in choosing between such pairs gives rise to several different strategies for computing global nilpotence bounds that trade simplicity of computation for decreased size of the bounds.

The following is by far the simplest formula, but yields very large bounds. We restrict ourselves to the case $\Gamma=\mathscr{A}_{n}=\mathscr{A}(n+1, n, \ldots, 3,2,1)$.

Theorem I.3. $H^{*}\left(\mathscr{A}_{n}\right)$ has global nilpotence bound $2^{((n+1)(n+2)(n+3) / 2)}$.

The next method of computation yields the best possible bounds that are attainable by the methods of Theorem I.1, but does so at the expense of requiring extensive calculations to compute most bounds.

For any $k, m$-allowable $\Gamma$ (Definition II.4) define integers:

$$
w_{0}=\left\{\begin{array}{ll}
2^{n_{m}-n_{k}-k} & \text { if } n_{m} \geq n_{k}+k \\
1 & \text { if } n_{m}<n_{k}+k
\end{array} \text { and } \quad w_{1}= \begin{cases}1 & \text { if } n_{m} \geq n_{k}+k \\
2^{n_{k}+k-n_{m}} & \text { if } n_{m}<n_{k}+k\end{cases}\right.
$$

Thus $h_{n_{k}, k}^{w_{0}} h_{n_{m}, m}^{w_{1}}=0$ in Proposition 3 .

Theorem I.4. For any finite Hopf subalgebra $\Gamma$,

$\operatorname{Bound}(\Gamma)=\left\{\begin{array}{l}1 \quad \text { if } H^{*}(\Gamma) \text { is nilfree, } \\ \min _{\substack{k, m \\ \Gamma, m \text {-allowable }}}\left\{w_{0} \cdot \operatorname{Bound}\left(\Gamma_{(k)}\right)+w_{1} \cdot \operatorname{Bound}\left(\Gamma_{(m)}\right)\right\} \text { otherwise, }\end{array}\right.$

is a global nilpotence for $H^{*}(\Gamma)$. 
A comparison of bounds for $H^{*}\left(\mathscr{A}_{n}\right)$ for $n \leq 6$ given by these two methods is shown in Table 1. The bounds given by Theorem I.4 are known to be the best possible for $H^{*}\left(\mathscr{A}_{0}\right)$ and $H^{*}\left(\mathscr{A}_{1}\right) . H^{*}\left(\mathscr{A}_{2}\right)$ must have a global nilpotence bound $\geq 4$ [11]. For $n \geq 3$ the global nilpotence bound for $H^{*}\left(\mathscr{A}_{n}\right)$ must be $\geq 2^{n+1}[5]$.

TABLE 1. Global nilpotence bounds for $H^{*}\left(\mathscr{A}_{n}\right)$ (scientific notation values rounded to 1 significant digit)

\begin{tabular}{lll}
$n$ & $\begin{array}{l}\text { Bound from } \\
\text { Theorem I.3 }\end{array}$ & $\begin{array}{l}\text { Bound from } \\
\text { Theorem I.4 }\end{array}$ \\
\hline 0 & 8 & 1 \\
1 & 4096 & 3 \\
2 & $1 E 9$ & 9 \\
3 & $1 E 18$ & 96 \\
4 & $4 E 31$ & 3,294 \\
5 & $4 E 50$ & 267,282 \\
6 & $7 E 75$ & $60,896,016$
\end{tabular}

The results in this paper constitute the major results of the author's Ph.D. thesis [10] under Donald Davis at Lehigh. The author is very grateful for the help and mathematical contributions of D. Anick, D. Davis, M. Hopkins, and G. Stengle. The helpful comments of the referee are also much appreciated.

\section{BACKGROUND AND NOTATION}

Let $R$ be a commutative algebra. An ideal $\mathscr{P} \subset R$ is prime if $\mathscr{P} \neq R$ and $x y \in P \Rightarrow x \in \mathscr{P}$ or $y \in \mathscr{P}$ for any $x, y \in R$. An ideal $\mathscr{Q}$ is primary if $\mathscr{Q} \neq R$ and $x y \in \mathscr{Q} \Rightarrow y \in \mathscr{Q}$ or $x^{k} \in \mathscr{Q}$ for some $k$. If $\mathscr{I}$ is any ideal then the radical of $\mathscr{I}$ is defined to be $r(\mathscr{I}) \equiv\left\{x \in R: x^{k} \in \mathscr{I}\right.$ for some $\left.k \in \mathbb{N}\right\}$. The radical of an ideal is an ideal. The radical of a primary ideal is prime. The radical of a prime ideal is the ideal itself. If $R$ is Noetherian, every ideal contains a power of its radical. In a Noetherian algebra every ideal is a finite intersection of primary ideals. Such a decomposition $\mathscr{I}=\bigcap_{i=1}^{n} \mathscr{Q}_{i}$ is said to be minimal if the $\mathscr{P}_{i}=r\left(\mathscr{Q}_{i}\right)$ are all distinct and $\mathscr{Q}_{i}$ does not contain $\bigcap_{j \neq i} \mathscr{Q}_{j}$ for any $i$ or $j$. Every ideal in a Noetherian ring has a minimal decomposition and the $\mathscr{P}_{i}$ are uniquely determined.

Let $\mathscr{A}^{*}$ be the dual of $\mathscr{A}$. Milnor [9] showed that $\mathscr{A}^{*} \simeq \mathbb{Z}_{2}\left[\xi_{1}, \xi_{2}, \xi_{3}, \ldots\right]$ with diagonal map $\psi: \mathscr{A}^{*} \rightarrow \mathscr{A}^{*} \otimes \mathscr{A}^{*}$ given by $\psi\left(\xi_{m}\right)=\sum_{a+b=m} \xi_{a}^{2^{b}} \otimes \xi_{b}$ on generators and extended to be an algebra homomorphism. For any subalgebra $\Gamma=\mathscr{A}\left(n_{1}, n_{2}, \ldots\right)$ of $\mathscr{A}$ we have

$$
\Gamma^{*} \simeq \mathbb{Z}_{2}\left[\xi_{1}, \xi_{2}, \xi_{3}, \ldots\right] /\left(\xi_{1}^{2_{1}}, \xi_{2}^{2^{n_{2}}}, \xi_{3}^{2^{n_{3}}}, \ldots\right) .
$$

We will say that $\xi_{i}^{j}=0$ in $\Gamma^{*}$ if the class of $\xi_{i}^{j}$ is zero in this quotient. For any graded $\mathbb{Z}_{2}$-algebra $\Gamma$ define the cohomology of $\Gamma$ to be $H^{*}(\Gamma) \equiv \operatorname{Ext}_{\Gamma}^{*}\left(\mathbb{Z}_{2}, \mathbb{Z}_{2}\right)$. 
This may be computed via the cobar resolution which is described fully in [1].

We now give some technical definitions that will be required in this paper.

Definition II.1. Suppose $\Gamma=\mathscr{A}\left(n_{1}, n_{2}, \ldots\right)$ is a finite Hopf subalgebra and $\Lambda$ is a normal Hopf subalgebra of $\Gamma$ such that $\Gamma / / \Lambda=E[\bar{x}]$ where $\bar{x}$ is the equivalence class of an indecomposable element $x \in \Gamma$. Let $\alpha \in H^{1, *}(\Gamma)$ be the class which is the image of the polynomial generator of $H^{*}(\Gamma / / \Lambda)$ under the map induced in cohomology by the quotient map. In this situation we say that $\Gamma$ is obtained from $\Lambda$ by the addition of one generator $\alpha$.

In this case, let $i: \Lambda \rightarrow \Gamma$ be the inclusion map and $i^{*}: H^{*, *}(\Gamma) \rightarrow H^{*, *}(\Lambda)$ the induced map.

Definition II.2. Suppose $\Gamma$ is a Hopf subalgebra of $\mathscr{A}$ for which $\xi_{t}^{2^{s-1}} \in \Gamma^{*}$ is primitive. In this case we define $h_{s, t}$ to be the corresponding class in $H^{1}(\Gamma)$.

Definition II.3. For any finite Hopf subalgebra $\Gamma=\mathscr{A}\left(n_{1}, n_{2}, \ldots\right)$ define

$$
\Gamma_{(j)}= \begin{cases}\mathscr{A}\left(n_{1}, \ldots, n_{j-1}, n_{j}-1, n_{j+1}, \ldots\right) & \text { if } n_{j} \neq 0 \\ \Gamma & \text { if } n_{j}=0 .\end{cases}
$$

Definition II.4. For any finite Hopf subalgebra $\Gamma=\mathscr{A}\left(n_{1}, n_{2}, \ldots\right)$ and any integers $k, m$ we say that $\Gamma$ is $k, m$-allowable iff

(a) $1 \leq k \leq m$;

(b) $n_{k}>0$ and $n_{m}>k$;

(c) $n_{k}>n_{i}$ and $n_{m}>n_{i}+k, \forall i<k$;

(d) $n_{k}>n_{i}+i-(k+m)$ and $n_{m}>n_{i}+i-m, \forall i<m$.

We will show in $\S \mathrm{IV}$ that if $\Gamma$ is $k, m$-allowable then $\Gamma_{(k)}$ and $\Gamma_{(m)}$ are Hopf subalgebras of $\Gamma$. A good reference for the background material in commutative algebra is [4]. Background on the Steenrod algebra and its subalgebras can be found in [8].

\section{THE EXISTENCE OF GLOBAL BOUNDS}

In this section we prove Theorem I.2. In order to do so we require the following result. Let $\Gamma=\mathscr{A}\left(n_{1}, \ldots, n_{k}\right)$ be any finite Hopf subalgebra of $\mathscr{A}$.

Theorem III.1 (Wilkerson [12]). $H^{*}(\Gamma)$ is Noetherian.

Theorem III.2. Any Noetherian algebra $R$ has bounded $x$-torsion $\forall x \in R$.

Proof. For any $x \in R$, define $\operatorname{Ann}(x)=\{y \in R: y x=0\}$. Consider the ascending chain of ideals:

$$
\operatorname{Ann}(x) \subseteq \operatorname{Ann}\left(x^{2}\right) \subseteq \operatorname{Ann}\left(x^{3}\right) \subseteq \cdots \subseteq \operatorname{Ann}\left(x^{n}\right) \subseteq \operatorname{Ann}\left(x^{n+1}\right) \subseteq \cdots .
$$

Since $R$ is Noetherian, this ascending chain must become stationary. Thus there is $m_{x}$ such that $\operatorname{Ann}\left(x^{k}\right)=\operatorname{Ann}\left(x^{m_{x}}\right)$ for all $k \geq m_{x}$ and $m_{x}$ is the least such integer. Hence, $x^{k} y=0 \Rightarrow x^{m_{x}} y=0$.

The following theorem was shown to the author by G. Stengle.

Theorem III.3. Every Noetherian algebra has a global torsion bound.

Proof. Let $R$ be Noetherian and (0) be the zero ideal in $R$. Then we have a minimal primary decomposition for $(0)$, i.e., $(0)=\bigcap_{i=1}^{n} \mathscr{Q}_{i}$ where the $\mathscr{Q}_{i}$ 
are primary ideals and $n \in \mathbb{N}$. Define $\mathscr{P}_{i}=r\left(\mathscr{Q}_{i}\right)$ for each $i \leq n$. Let $m_{i}$ be the smallest integer such that $\mathscr{P}_{i}^{m_{i}} \subset \mathscr{Q}_{i}$. Let $m=\max _{i}\left\{m_{i}\right\}$. Suppose $x^{k} y=0$. Then $x^{k} y \in(0)=\bigcap_{i=1}^{n} \mathscr{Q}_{i}$ and so $x^{k} y \in \mathscr{Q}_{i}$ for each $i \leq n$. Since $\mathscr{Q}_{i}$ is primary, $y \in \mathscr{Q}_{i}$ or $x^{k j} \in \mathscr{Q}_{i}$ for some $j$, which implies that $y \in \mathscr{Q}_{i}$ or $x \in \mathscr{P}_{i}$. Thus $y \in \mathscr{Q}_{i}$ or $x^{m} \in \mathscr{Q}_{i}$. So $x^{m} y \in \mathscr{Q}_{i}$ for any $i \leq n$. Hence $x^{m} y \in \bigcap_{i=1}^{n} \mathscr{Q}_{i}=(0)$. So $x^{m} y=0$.

Theorem III.4. Every Noetherian algebra $R$ has a global nilpotence bound.

Proof. This is an obvious corollary to the previous theorem (take $y=1$ ). But it has a simpler proof. Since $R$ is Noetherian the ideal $(0)$ must contain some power of $r(0)$, which is just the set of nilpotents in $R$.

Theorem III.5. In any Noetherian algebra the smallest global torsion bound and the smallest global nilpotence bound are equal.

Proof. As in the proof of Theorem III.3, let $R$ be Noetherian and (0) be the zero ideal in $R$. Then we have a minimal primary decomposition for $(0)$, i.e. $(0)=\bigcap_{i=1}^{n} \mathscr{Q}_{i}$ where the $\mathscr{Q}_{i}$ are primary ideals and $n \in \mathbb{N}$. Define $\mathscr{P}_{i}=r\left(\mathscr{Q}_{i}\right)$ for each $i \leq n$. Let $m_{i}$ be the smallest integer such that $\mathscr{P}_{i}^{m_{i}} \subset \mathscr{Q}_{i}$. Let $m=\max _{i}\left\{m_{i}\right\}$. We first show that $m$ is the smallest global torsion bound for $R$. If $m=1$ it is clearly minimal. If $m>1$ then since $m_{j}=m$ for some $j$, we can choose $z \in \mathscr{P}_{j}$ so that $z^{m} \in \mathscr{Q}_{j}$ and $z^{m-1} \notin \mathscr{Q}_{j}$. Since our decomposition is minimal there is $w \in \bigcap_{i \neq j} \mathscr{Q}_{i}$ such that $w \notin \mathscr{Q}_{j} \subseteq \mathscr{P}_{j}$. Then $z^{m-1} w \notin \mathscr{Q}_{j}$ since $z^{m-1} \notin \mathscr{Q}_{j}$ and $w \notin \mathscr{P}_{j}$ and $\mathscr{Q}_{j}$ is primary. So $m$ is the smallest global torsion bound.

Now let $b=$ the smallest global nilpotence bound. Clearly $m \geq b$ since $x^{k}=0 \Rightarrow x^{k} \cdot 1=0$. It follows that if $m=1$ then $m=b=1$. If $m>1$ then since $z^{m} w=0$ we have $(z w)^{m}=\left(z^{m} w\right) w^{m-1}=0 w=0$. But $(z w)^{m-1}=$ $\left(z^{m-1} w\right) w^{m-2}$ (where $w^{0}=1$ ). Further, $z^{m-1} w \notin \mathscr{Q}_{j}$ and $w^{m-2} \notin \mathscr{P}_{j}$ (because $r\left(\mathscr{P}_{j}\right)=\mathscr{P}_{j}$ and $\left.w \notin \mathscr{P}_{j}\right)$, their product $(z w)^{m-1} \notin \mathscr{Q}_{j}$ because $\mathscr{Q}_{j}$ is primary. Thus $(z w)^{m-1} \notin \bigcap_{i=1}^{n} \mathscr{Q}_{i}=(0) \Rightarrow(z w)^{m-1} \neq 0$. So $z w$ has nilpotence $m$. Thus $b \geq m$. So $m=b$.

Thus the question of finding a global torsion bound is equivalent to that of finding the global nilpotence bound. Hence we now have

Proof (of Theorem I.2). By Theorem III.1 $H^{*}(\Gamma)$ is Noetherian. Thus by Theorem III. 3 it has a global torsion bound, and hence a smallest one. By Theorem III.5 this is equal to the smallest global nilpotence bound. The theorem follows.

Corollary III.1. The bounds computed by Theorem I.1 are also global torsion bounds.

\section{COMPUTING GLOBAL BOUNDS}

In this section we give the proof of Theorem I.1. It suffices to prove Theorem I.4 since this satisfies the existence requirement of Theorem I.1. We begin by showing how Theorem I.4 follows from Propositions 1-3.

Proof (of Theorem I.4). For any finite Hopf subalgebra $\Gamma=\mathscr{A}\left(n_{1}, n_{2}, \ldots\right)$ define $\sigma(\Gamma)=\sum_{i} n_{i}$. Notice that $\Gamma$ finite $\Rightarrow \sigma(\Gamma)<\infty$. We proceed by 
induction on $\sigma(\Gamma)$. If $\sigma(\Gamma)=1$ then $\Gamma$ is exterior and $H^{*}(\Gamma)$ is nilfree by Proposition II.1. Hence $\operatorname{Bound}(\Gamma)=1$ and this is trivially a global nilpotence/torsion bound for $H^{*}(\Gamma)$. So assume that $\operatorname{Bound}(\Lambda)$ is a global nilpotence/torsion bound for the cohomology of any $\Lambda$ with $\sigma(\Lambda)<\sigma(\Gamma)$. If $\Gamma$ has nilfree cohomology, then $\operatorname{Bound}(\Gamma)=1$ and is still trivially a global nilpotence/torsion bound. If not, then by Proposition 3 there is at least one pair of integers $k, m$ so that $\Gamma$ is obtained from $\Gamma_{(k)}$ (resp. $\left.\Gamma_{(m)}\right)$ by the addition of one generator $\alpha_{k}$ (resp. $\alpha_{m}$ ) so that $\alpha_{k}^{w_{0}} \alpha_{m}^{w_{1}}=0$. By the induction hypothesis, Bound $\left(\Gamma_{(i)}\right)$ is a global nilpotence/torsion bound for $H^{*}\left(\Gamma_{(i)}\right)$ for any $i$ such that $\Gamma_{(i)}$ is a proper subalgebra (i.e., $n_{i} \neq 0$ ), since then $\sigma\left(\Gamma_{(i)}\right)<\sigma(\Gamma)$. Thus by Proposition 2, $H^{*}(\Gamma)$ has global nilpotence/torsion bound $w_{0} \cdot \operatorname{Bound}\left(\Gamma_{(k)}\right)+w_{1} \cdot \operatorname{Bound}\left(\Gamma_{(m)}\right)$. Since this will be true for any choice of the pair $k, m$ such that $\Gamma$ is $k, m$-allowable, we can take the minimum of all such values to get the best possible value using these methods. This is precisely what $\operatorname{Bound}(\Gamma)$ is, completing the induction and hence the proof.

So we must now prove Propositions 1-3. Proposition 1 was proved by W. H. Lin [6].

In order to prove Proposition 2 we require the following result of Lin [6].

Theorem IV.1. If $\Gamma$ is obtained from $\Lambda$ by the addition of one generator whose class is $\alpha$ and $i^{*}: H^{*, *}(\Gamma) \rightarrow H^{*, *}(\Lambda)$ is the map induced by the inclusion, then $\operatorname{Ker}\left(i^{*}\right)=$ ideal of $H^{*}(\Gamma)$ generated by $\alpha$.

Using this result we can now prove Proposition 2, which is the key result used in this method of computing global nilpotence/torsion bounds.

Proof (of Proposition 2). Let $x \in H^{*}(\Gamma)$ be nilpotent. Let $i_{j}: \Lambda_{j} \rightarrow \Gamma$ be the inclusion map and let $M=\sum_{j=1}^{n} w_{j} m_{j}$. Then $x$ nilpotent $\Rightarrow x^{k}=0$ for some $k \Rightarrow i_{j}^{*}(x)^{k}=i_{j}^{*}\left(x^{k}\right)=0$ for each $j=1, \ldots, n \Rightarrow i_{j}^{*}(x)$ is nilpotent for each $j \Rightarrow i_{j}^{*}(x)^{m_{j}}=0$ for each $j \Rightarrow i_{j}^{*}\left(x^{m_{j}}\right)=0$ for each $j \Rightarrow x^{m_{j}} \in \operatorname{Ker} i_{j}^{*}$ for each $j \Rightarrow x^{m_{j}}=\alpha_{j} \cdot y_{j}$ for each $j$ and some $y_{j} \in H^{*}(\Gamma)$ (by Theorem IV.1) $\Rightarrow x^{M}=\prod_{j=1}^{n}\left(x^{m_{j}}\right)^{w_{j}}=\left(\prod_{j=1}^{n} \alpha_{j}^{w_{j}}\right) \cdot y=0 \cdot y=0$ for some $y \in \Gamma$.

It should be noted at this point that we will only require the cases $n=1,2$ for our purposes.

It now remains to prove Proposition 3. In doing so we imitate the methods of Lin [6]. We proceed by a series of lemmas. Throughout we will let $\Gamma=$ $\mathscr{A}\left(n_{1}, n_{2}, \ldots\right)$ be any finite Hopf subalgebra of $\mathscr{A}$.

Lemma IV.1. $h_{s, t}$ is a well-defined nonzero element of $H^{*}(\Gamma)$ if $n_{i}+i-t+1 \leq$ $s \leq n_{t}$ for all $i<t$.

Proof. Since $\left[\xi_{t}^{2^{s-1}}\right]$ cannot be a coboundary it is enough to show it is a cocycle. But $d\left[\xi_{t}^{2^{s-1}}\right]=\sum_{i<t}\left[\xi_{i}^{2^{t-i+s-1}} \mid \xi_{t-i}^{2^{s-1}}\right]=0$ since $n_{i} \leq t-i+s-1 \Rightarrow \xi_{i}^{2^{t-i+s-1}}$ is zero in $\Gamma^{*}=\mathscr{A}\left(n_{1}, n_{2}, \ldots\right)^{*}$.

For any rational number $q$ let $\llbracket q \rrbracket$ be the greatest integer less than or equal to $q$. There are Steenrod operations in cohomology [7].

Lemma IV.2. There are Steenrod operations on $H^{*, *}(\Gamma)$ satisfying:

1. $S q^{i}: H^{s, t}(\Gamma) \rightarrow H^{s+i, 2 t}(\Gamma)$, 
2. $S q^{i}(a b)=\sum_{j+k=i} S q^{j}(a) S q^{k}(b)$,

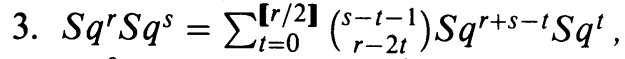

4. $S q^{0}\left(\left[\alpha_{1}|\cdots| \alpha_{n}\right]\right)=\left[\alpha_{1}^{2}|\cdots| \alpha_{n}^{2}\right]$,

5. $S q^{s}(x)=x^{2}$ if $x \in H^{s, *}(\Gamma)$,

6. $S q^{s}(x)=0$ if $x \in H^{r, *}(\Gamma)$ and $r<s$.

Thus by property 4 we have $S q^{0}\left(h_{s, t}\right)=h_{s+1, t}$ whenever these are defined.

Lemma IV.3. Let $x, y$ be nonzero elements of $H^{1, *}(\Gamma)$.

Define $Q_{b}=S q^{2^{b-1}} S q^{2^{b-2}} \cdots S q^{4} S q^{2} S q^{1}$. Then

$$
Q_{b}(x y)=\left(x^{2^{b}}\right)\left(\left(S q^{0}\right)^{b} y\right)+\left(\left(S q^{0}\right)^{b} x\right)\left(y^{2^{b}}\right) .
$$

Proof. This is proved in [6]. There is an easy proof using Lemma IV.2.

Lemma IV.4. If $\Gamma$ is $k$, m-allowable then

(a) $h_{n_{k}+k, m}$ and $h_{n_{k}, k}$ are well-defined nonzero elements of $H^{1}(\Gamma)$ if $n_{m} \geq$ $n_{k}+k$, and

(b) $h_{n_{m}, m}$ and $h_{n_{m}-k, k}$ are well-defined nonzero elements of $H^{1}(\Gamma)$ if $n_{m}<$ $n_{k}+k$.

Proof. Case a: $n_{m} \geq n_{k}+k$. Then $\Gamma k, m$-allowable $\Rightarrow n_{i}+i-k<n_{i}<n_{k}$ for $i<k \Rightarrow h_{n_{k}, k} \neq 0 \in H^{1}(\Gamma)$ by Lemma IV.1, and $\Gamma$ is $k, m$-allowable $\Rightarrow n_{i}+i-m<n_{k}+k \leq n_{m}$ for $i<m \Rightarrow h_{n_{k}+k, m} \neq 0 \in H^{1}(\Gamma)$ by Lemma IV.1.

Case b: $n_{m}<n_{k}+k$. Then $\Gamma$ is $k, m$-allowable $\Rightarrow n_{i}+i-k<n_{i}<$ $n_{m}-k<n_{k}$ for $i<k \Rightarrow h_{n_{m}-k, k} \neq 0 \in H^{1}(\Gamma)$ by Lemma IV.1, and $\Gamma$ is $k, m$-allowable $\Rightarrow n_{i}+i-m<n_{m}$ for $i<m \Rightarrow h_{n_{m}, m} \neq 0 \in H^{1}(\Gamma)$ by Lemma IV.1.

Lemma IV.5. If $\Gamma$ is $k$, $m$-allowable then

(a) $h_{n_{k}+k, m} h_{n_{k}, k}=0$ in $H^{*}(\Gamma)$ if $n_{m} \geq n_{k}+k$, and

(b) $h_{n_{m}, m} h_{n_{m}-k, k}=0$ in $H^{*}(\Gamma)$ if $n_{m}<n_{k}+k$.

Proof. Case a: $n_{m} \geq n_{k}+k$. Then $\Gamma$ is $k, m$-allowable $\Rightarrow n_{i}<n_{k}$ for $i<$ $k \Rightarrow \xi_{i}^{2^{n_{k}-1}}=0$ in $\Gamma^{*}$ for $i<k$, and $\Gamma$ is $k, m$-allowable $\Rightarrow n_{j}<n_{k}+k+m-j$ for $j<m \Rightarrow \xi_{j}^{2^{n_{k}+k+m-j-1}}=0$ in $\Gamma^{*}$ for $j<m$. Thus in the cobar resolution for $H^{*}(\Gamma)$ :

$$
\begin{aligned}
d\left[\xi_{k+m}^{2_{k}-1}\right] & =\sum_{i=1}^{k+m-1}\left[\xi_{k+m-i}^{2^{n_{k}+i-1}} \mid \xi_{i}^{2^{n_{k}-1}}\right] \\
& =\sum_{i<k}\left[\xi_{k+m-i}^{n^{n_{k}+i-1}} \mid \xi_{i}^{2^{n_{k}-1}}\right]+\sum_{j<m}\left[\xi_{j}^{2^{n_{k}+k+m-j-1}} \mid \xi_{k+m-j}^{2^{n_{k}-1}}\right]+\left[\xi_{m}^{2^{n_{k}+k-1}} \mid \xi_{k}^{2^{n_{k}-1}}\right] \\
& =\left[\xi_{m}^{2^{n_{k}+k-1}} \mid \xi_{k}^{2^{n_{k}-1}}\right] .
\end{aligned}
$$

Hence, $h_{n_{k}+k, m} h_{n_{k}, k}=0$ in $H^{*}(\Gamma)$.

Case b: $n_{m}<n_{k}+k$. Then $\Gamma$ is $k, m$-allowable $\Rightarrow n_{i}<n_{m}-k$ for $i<$ $k \Rightarrow \xi_{i}^{2^{n_{m}-k-1}}=0$ in $\Gamma^{*}$ for $i<k$, and $\Gamma$ is $k, m$-allowable $\Rightarrow n_{j}<n_{m}+m-j$ for $j<m \Rightarrow \xi_{j}^{2^{n_{m+m-j-1}}}=0$ in $\Gamma^{*}$ for $j<m$. Thus in the cobar resolution for 
$H^{*}(\Gamma)$ :

$$
\begin{aligned}
d\left[\xi_{k+m}^{2^{n_{m}-k-1}}\right]= & \sum_{i=1}^{k+m-1}\left[\xi_{k+m-i}^{2^{n_{m}+i-k-1}} \mid \xi_{i}^{2^{n_{m}-k-1}}\right] \\
= & \sum_{i<k}\left[\xi_{k+m-i}^{2^{n_{m}+-k-1}} \mid \xi_{i}^{2^{n_{m}-k-1}}\right] \\
& +\sum_{j<m}\left[\xi_{j}^{2^{n_{k}+m-j-1}} \mid \xi_{k+m-j}^{n_{m}-k-1}\right]+\left[\xi_{m}^{2^{n_{m}-1}} \mid \xi_{k}^{2^{n_{m}-k-1}}\right] \\
= & {\left[\xi_{m}^{2_{m}-1} \mid \xi_{k}^{2^{n_{m}-k-1}}\right] . }
\end{aligned}
$$

Hence, $h_{n_{m}, m} h_{n_{m}-k, k}=0$ in $H^{*}(\Gamma)$.

Lemma IV.6. If $\Gamma$ is $k$, m-allowable then

(a) $h_{n_{m}, m} h_{n_{k}, k}^{2_{m}-n_{k}-k}=0$ in $H^{*}(\Gamma)$ if $n_{m} \geq n_{k}+k$, and

(b) $h_{n_{m}, m}^{2^{n_{k}+k-n_{m}}} h_{n_{k}, k}=0$ in $H^{*}(\Gamma)$ if $n_{m}<n_{k}+k$.

Proof. Notice that by definition $h_{n_{t}+b, t}=0$ in $H^{*}(\Gamma)$ for any $t$ if $b>0$.

Case a: $n_{m} \geq n_{k}+k$. If $n_{m}=n_{k}+k$ then we are done by Lemma IV.5.

So assume $n_{m}>n_{k}+k$ and let $b=n_{m}-n_{k}-k$. Then by Lemma IV.3 and Lemma IV.4,

$$
\begin{aligned}
0 & =Q_{b}(0)=Q_{b}\left(h_{n_{k}+k, m} h_{n_{k}, k}\right) \\
& =h_{n_{k}+k, m}^{2^{b}} h_{n_{k}+b, k}+h_{n_{k}+k+b, m} h_{n_{k}, k}^{2^{b}}=h_{n_{m}, m} h_{n_{k}, k}^{2^{n_{m}-n_{k}-k}} .
\end{aligned}
$$

Case b: $n_{m}<n_{k}+k$. Let $b=n_{m}-n_{k}-k$. Then by Lemma IV.3 and Lemma IV.4,

$$
\begin{aligned}
0 & =Q_{b}(0)=Q_{b}\left(h_{n_{m}, m} h_{n_{m}-k, k}\right) \\
& =h_{n_{m}, m}^{2^{b}} h_{n_{m}-k+b, k}+h_{n_{m}+b, m} h_{n_{m}-k, k}^{2^{b}}=h_{n_{m}, m}^{2^{n_{k}+k-n_{m}}} h_{n_{k}, k} .
\end{aligned}
$$

Lemma IV.7. If $\Gamma_{(i)}$ is a proper Hopf subalgebra of $\Gamma$ then $\Gamma$ is obtained from $\Gamma_{(i)}$ by the addition one generator $h_{n_{i}, i}$.

Proof. Since $\Gamma_{(i)}$ is a proper subalgebra $n_{i}>0$. Let $\Lambda=\Gamma_{(i)}$ and $q=n_{i}-1$. We must show $\Lambda$ is normal in $\Gamma$, i.e., that $\Gamma \cdot \bar{\Lambda}=\bar{\Lambda} \cdot \Gamma$ where $\bar{\Lambda}$ is the augmentation ideal. To accomplish this we imitate the methods of Margolis [8]. It suffices to show that

$$
\mathscr{C}=\mathbb{Z}_{2} \text { vector space span of }\left\{S q\left(r_{1}, \ldots\right) \in \Gamma \mid S q\left(r_{1}, \ldots\right) \neq P_{i}^{q}\right\}
$$

spans both $\Gamma \cdot \bar{\Lambda}$ and $\bar{\Lambda} \cdot \Gamma$. If $x$ and $y$ are Milnor basis elements then $P_{i}^{q}$ is a summand of $x y$ if and only if $x=P_{j}^{q+i-j}$ and $y=P_{i-j}^{q}$ for some $j$ [8]. By hypothesis $\Lambda$ is a Hopf subalgebra of $\Gamma$ and so either $n_{j} \leq q+i-j$ or $n_{i-j} \leq q$ for any $j<i$. Thus $P_{j}^{q+i-j}$ and $P_{i-j}^{q}$ are not in $\Gamma$ for any $j<i$. Thus for any Milnor elements $x, y \in \Gamma, P_{i}^{q}$ is not a summand of $x y$ or $y x$ unless $x=1$ or $y=1$. so $\Gamma \cdot \bar{\Lambda} \subset \mathscr{C}$ and $\bar{\Lambda} \cdot \Gamma \subset \mathscr{C}$. To obtain the opposite inclusions we 
note that as shown in [8], for any Milnor element $S q\left(r_{1}, \ldots\right) \in \mathscr{C}$,

$$
\begin{aligned}
S q\left(r_{1}, \ldots\right)= & S q\left(r_{1}, \ldots, r_{j}-2^{h}, \ldots\right) \cdot P_{j}^{h} \\
& + \text { terms of lower excess than } S q\left(r_{1}, \ldots\right) \\
= & P_{j}^{h} \cdot S q\left(r_{1}, \ldots, r_{j}-2^{h}, \ldots\right) \\
& + \text { terms of lower excess than } S q\left(r_{1}, \ldots\right) .
\end{aligned}
$$

Notice that here that $P_{i}^{q}$ is not one of the terms of lower excess (nor is it $S q\left(r_{1}, \ldots\right)$ since this is in $\left.\mathscr{C}\right)$ because if it were it would be a summand of the product $S q\left(r_{1}, \ldots, r_{j}-2^{h}, \ldots\right) \cdot P_{j}^{h}\left(\right.$ resp. $\left.P_{j}^{h} \cdot S q\left(r_{1}, \ldots, r_{j}-2^{h}, \ldots\right)\right)$ which we have already shown to be impossible since neither factor is 1 . Hence by induction on excess, $S q\left(r_{1}, \ldots\right) \in \mathscr{C} \Rightarrow S q\left(r_{1}, \ldots\right) \in \Gamma \cdot \bar{\Lambda}$ and $S q\left(r_{1}, \ldots\right) \in$ $\bar{\Lambda} \cdot \Gamma$. So $\mathscr{C} \subset \bar{\Lambda} \cdot \Gamma$ and $\mathscr{C} \subset \Gamma \cdot \bar{\Lambda}$ and so $\Lambda$ is normal in $\Gamma$. Thus the quotient $\Gamma / / \Lambda=\Gamma / \Gamma \cdot \bar{\Lambda}=E\left[P_{i}^{q}\right]$ is thus well defined and so $\Gamma$ is obtained from $\Lambda$ by the addition of one generator whose cobar representative is $\left[\xi_{i}^{2^{q}}\right]=\left[\xi_{i}^{2^{n_{i}-1}}\right]$, i.e., $h_{n_{i}, i}$.

Lemma IV.8. If $\Gamma$ is $k$, m-allowable then $\Gamma_{(k)}$ and $\Gamma_{(m)}$ are Hopf subalgebras of $\Gamma$.

Proof. Let

$$
\begin{gathered}
\Gamma=\mathscr{A}\left(n_{1}, n_{2}, \ldots\right) \\
\Gamma_{(k)}=\mathscr{A}\left(p_{1}, p_{2}, \ldots\right)=\mathscr{A}\left(n_{1}, n_{2}, \ldots, n_{k}-1, \ldots\right),
\end{gathered}
$$

and

$$
\Gamma_{(m)}=\mathscr{A}\left(q_{1}, q_{2}, \ldots\right)=\mathscr{A}\left(n_{1}, n_{2}, \ldots, n_{m}-1, \ldots\right) .
$$

Since $\Gamma$ is a Hopf subalgebra of $\mathscr{A}$ we have either $n_{v} \geq n_{u}+u-v$ or $n_{v} \geq n_{v-u}$ for all $1 \leq u<v$, and must show this property holds for both the $p_{i}$ and the $q_{i}$.

For $\Gamma_{(k)}$, the lemma easily follows from the following facts. If $v<k$ then $p_{i}=n_{i}$ for all $i \leq v$. If $v=k$ then $p_{k}=n_{k}-1 \geq n_{u} \geq n_{u}+(u-k)$ for all $u<v$ since $\Gamma$ is $k, m$-allowable. Finally, if $v>k$ then $p_{u} \leq n_{u}$ for all $u<v$ and $p_{v}=n_{v}$. Similarly for $\Gamma_{(m)}$, the lemma easily follows from the following facts. If $v<m$ then $q_{i}=n_{i}$ for all $i \leq v$. If $v=m$ then $q_{m}=n_{m}-1 \geq n_{u}+(u-m)$ for all $u<v$ since $\Gamma$ is $k, m$-allowable. Finally, if $v>m$ then $q_{u} \leq n_{u}$ for all $u<v$ and $q_{v}=n_{v}$.

Using these results we can now prove Proposition 3.

Proof (of Proposition 3). $\Gamma$ is $k, m$-allowable $\Rightarrow \Gamma_{(k)}$ (and $\Gamma_{(m)}$ ) are Hopf subalgebras of $\Gamma$ (by Lemma IV.8) which $\Gamma$ is obtained from by the addition of one generator whose class is $h_{n_{k}, k}$ (resp. $h_{n_{m}, m}$ ) (by Lemma IV.7). The relations (a) and (b) in the proposition are then true by Lemma IV.6. Thus it suffices to show that for any $\Gamma=\mathscr{A}\left(n_{1}, n_{2}, \ldots\right)$ which is not of type (a) of Proposition 1, there is one pair of integers $k, m$ such that $\Gamma$ is $k, m$-allowable. Let $k=\min \left\{j: n_{j} \neq 0\right\}$ and let $m=\min \left\{j: n_{j}>k\right\}$ (which must exist since $\Gamma$ is not of type (a) of Proposition 1). We now show that $\Gamma$ is $k, m$ allowable (refer to Definition II.4). Conditions (a) and (b) follow trivially from the definition of $k$ and $m$. By definition of $k, n_{i}=0$ for $i<k$, so condition 
(c) follows. Finally, by definition of $m, n_{i} \leq k$ for $i<m$ and $n_{m}>k$ so $n_{m}>k \geq n_{i}>n_{i}+i-m$ for $i<m$ and $n_{k}>0 \geq n_{i}-k>n_{i}-k+i-m$ for $i<m$.

All that remains is to prove Theorem I.3.

Proof (of Theorem I.3). Let $n \in \mathbb{N}$ and $W=2^{n+2}$. If we write $\mathscr{A}_{n}=$ $\mathscr{A}\left(n_{1}, n_{2}, \ldots, n_{n+1}=\mathscr{A}(n+1, n, \ldots, 2,1)\right.$ then $n_{i}=n+2-i$ for $1 \leq$ $i \leq n+1$. Then $w_{i} \leq 2^{\left|n_{m}-n_{k}-k\right|} \leq 2^{n_{k}+k} \leq 2^{(n+2-k)+k}=2^{n+2}=W$ for all $w_{i}$ involved in the calculation of $\operatorname{Bound}\left(\mathscr{A}_{n}\right)$ in Theorem I.4. For any finite Hopf subalgebra of $\mathscr{A}_{n}, \Gamma=\mathscr{A}\left(n_{1}, n_{2}, \ldots\right)$, define Toobig $(\Gamma)=(2 W)^{\sigma(\Gamma)}=$ $2^{(n+3) \sigma(\Gamma)}$ where $\sigma(\Gamma)=\sum_{i} n_{i}$. Assume towards induction that $\operatorname{Toobig}(\Lambda) \geq$ $\operatorname{Bound}(\Lambda)$ for any $\Lambda$ with $\sigma(\Lambda)<\sigma(\Gamma)$. Then Toobig $(\Gamma) \geq \operatorname{Bound}(\Gamma)$ since

$$
\begin{aligned}
\operatorname{Bound}(\Gamma) & \leq \min _{\beta(k, m, \Gamma)=1}\left\{w_{0} \cdot \operatorname{Bound}\left(\Gamma_{(k)}\right)+w_{1} \cdot \operatorname{Bound}\left(\Gamma_{(m)}\right)\right\} \\
& \leq 2 \cdot W \cdot \operatorname{Toobig}\left(\Gamma_{(i)}\right), \quad \forall i \text { with } \Gamma_{(i)} \neq \Gamma \\
& \leq 2 \cdot W \cdot\left(2^{(n+3) \sigma\left(\Gamma_{(i)}\right)}\right)=2^{(n+3)\left(\sigma\left(\Gamma_{(i)}\right)+1\right)} \\
& =2^{(n+3) \sigma(\Gamma)}=\operatorname{Toobig}(\Gamma) .
\end{aligned}
$$

Taking $\Gamma=\mathscr{A}_{n}$, we have $\sigma(\Gamma)=(n+1)(n+2) / 2$ and so Toobig $\left(\mathscr{A}_{n}\right)=$ $2^{((n+1)(n+2)(n+3) / 2)}$ is a global nilpotence bound for $H^{*}\left(\mathscr{A}_{n}\right)$.

\section{BIBLIOGRAPHY}

1. J. F. Adams, On the nonexistence of elements of Hopf invariant one, Ann. of Math. 62 (1960), 20-104.

2. J. F. Adams and H. R. Margolis, Sub-Hopf-algebras of the Steenrod algebra, Proc. Cambridge Philos. Soc. 76 (1974), 45-52.

3. D. W. Anderson and D. M. Davis, A vanishing theorem in homological algebra, Comment. Math. Helv. 48 (1973), 318-327.

4. M. Atiyah, Introduction to commutative algebra, Addison-Wesley, Reading, Mass., 1969.

5. D. M. Davis, An infinite family in the cohomology of the Steenrod algebra, J. Pure Appl. Algebra 21 (1981), 145-150.

6. W. H. Lin, Cohomology of sub-Hopf-algebras of the Steenrod algebra, J. Pure Appl. Algebra 10 (1977), 101-113.

7. A. Liulevicius, The factorization of cyclic reduced powers by secondary cohomology operations, Mem. Amer. Math. Soc., No. 42, 1962.

8. H. R. Margolis, Spectra and the Steenrod algebra, North-Holland Math Library, vol. 29, North-Holland, Amsterdam, 1983.

9. J. Milnor, The Steenrod algebra and its dual, Ann. of Math. 67 (1958), 150-171.

10. K. G. Monks, Nilpotence and torsion in the Steenrod algebra and its cohomology, Ph.D. thesis, Lehigh University, 1989.

11. N. Shimada and A. Iwai, On the cohomology of some Hopf algebras, Nagoya Math J. 30 (1967), 103-111.

12. C. Wilkerson, The cohomology algebras of finite dimensional Hopf algebras, Trans. Amer. Math. Soc. 264 (1981), 137-150.

Department of Mathematics, University of Scranton, Scranton, Pennsylvania 18510 E-mail address: kgm303@jaguar.uofs.edu 